\title{
Optical Activity of an Asymmetric Substance
}

\author{
Frida U. Ermawati* \\ Physics Deptartment Surabaya State University \\ Ketintang Campus, Surabaya 60231
}

\begin{abstract}
This paper reported the investigation on the property of optical activity from three different isomers of L-, Dand DL- $\alpha$-alanine $\left(\mathrm{CH}_{3} \mathrm{CHNH}_{2} \mathrm{CO}_{2} \mathrm{H}\right)$, a proposed radiation dosimetric material. The aim was to confirm the impact of attachment of $\alpha$-carbon atom of the systems to the four different groups, that is, the $\beta$-carbon or methyl group, $\mathrm{H}$-group, $\mathrm{CO}_{2} \mathrm{H}$-group and $\mathrm{NH}_{2}$-group, to their response to any incoming plane-polarized light. Optical activity of a substance is a measure of the ability of a substance to rotate a plane of polarization, if solution of the substance is placed in the path of plane-polarized light. A polarimeter of Perkin-Elmer Model 141 with a Sodium lamp of 584-nm wavelength (D-line) and equipped with a digital counter was occupied in this work to measure the number of degrees of rotation of plane-polarized light at room temperature due to $8.3 \%$ concentration of each L-, D- and DL- $\alpha$-alanine solutions. It was obtained that molecules of L-isomer rotate the counter of the polarimeter clockwise, from the observer's point a view. While the molecules of D-isomer rotate the counter anti clockwise, at the similar amount of rotation. These results confirm the asymmetric structure belong to the $\alpha$-alanine system. Molecules of DL-isomer, however, do not rotate the counter at all. The existence of similar number of the D- and L-molecules in the DL-solution is the cause.
\end{abstract}

KEYWORDS: optic active, polarimeter,a symmetric solution

\section{INTRODUCTION}

$\alpha$-Alanine (see Figure-1a) is an important substance in radiation dosimetry area because $\alpha$-alanine has been proposed as a dosimetric material for couple of tens years. Panta et al.[1, 2] showed that typical features of $\alpha$-alanine such as elemental chemical composition, electron density, effective atomic number and specific gravity of alanine are very close to those of soft biological tissue and water, it also providing similarity to biological systems or water-equivalent systems in terms of absorption of radiation energy. Regulla and Deffner[3] found that deamination of an $\alpha$-alanine molecule at room temperature produces a stable alkyl free radical $\left(\mathrm{CH} 3 \mathrm{C}^{\bullet} \mathrm{HCOO}\right)$. Sagstuen et al.,[4, 5] even found the existence of several radicals simultaneously in the system. Deamination can be induced in solid or aqueous $\alpha$-alanine by radiation. When normal $\alpha$-alanine is irradiated, the radiation causes certain $\alpha$ alanine molecules to become free radicals, and as these radicals are stable, the free radical content can later be measured in order to find out how much radiation the $\alpha$-alanine was exposed to. In this way, one can be assured that complex radiotherapy treatment plans will deliver the intended pattern of radiation dose.

Due to the significant role of $\alpha$-alanine above, it is therefore important to investigate many aspects of this particular substance, including their property of optical activity. Thus, this paper is intended to report the work on optical activity property from three different isomers of L-, D- and DL- $\alpha$-alanine. The aim was to confirm the impact of attachment of $\alpha$-carbon atom of the systems to the four different groups (see again

*E-MAIL: frida_dua@yahoo.com

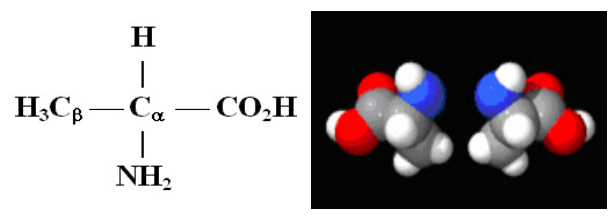

(a)

FIG. 1: (a) Structure of $\alpha$-alanine: $\alpha$-carbon atom attaches to the four different groups, i.e. $\beta$-carbon $\left(\mathrm{CH}_{3-}\right), \mathrm{H}-, \mathrm{CO}_{2} \mathrm{H}-$ and $\mathrm{NH}_{2-}$ groups, respectively [1]. (b) Atomic arrangements of L- and D- $\alpha$ alanine: $\mathrm{C}$ atom=Grey color, $\mathrm{H}=$ white, $\mathrm{O}=$ red and $\mathrm{N}=$ blue [8] These arrangements are called as an asymmetric arrangement. Detail discussion on this structure is given in the text. See also Figure 4 for clarity.

Figure-1a) to their response to any incoming plane-polarized light.

\section{THEORETICAL BACKGROUND}

\section{A. $\quad \alpha$-Alanine: Sources, Chemical Synthesis and Molecular Structure}

$\alpha$-Alanine [6] (abbreviated as Ala or A) is an $\alpha$-amino acid with the chemical formula $\mathrm{HO}_{2} \mathrm{CCH}\left(\mathrm{NH}_{2}\right) \mathrm{CH}_{3}$ (see Figure1a). $\alpha$-Alanine is a nonessential amino acid, meaning it can be manufactured by the human body and does not need to be obtained directly through the diet. However, chemical synthesis on $\alpha$-alanine can also be carried out via the condensation of acetaldehyde with ammonium chloride in the presence of potassium cyanide by the Strecker reaction [7].

As seen in Figure-1a, $\alpha$-carbon atom of alanine is bound to four different groups, namely $\beta$-carbon group or methyl group 
$\left(-\mathrm{CH}_{3}\right), \mathrm{H}$-group, $\mathrm{CO}_{2} \mathrm{H}$ group and $\mathrm{NH} 2$ group. Attachment of the $\alpha$-carbon atom to the methyl group making it one of the simplest $\alpha$-amino acids with respect to molecular structure and hence resulting in $\alpha$-alanine being classified as an aliphatic amino acid. The methyl group of $\alpha$-alanine is nonreactive and thus, almost never directly involved in protein function.

\section{B. Polarimetry, Measuring optical rotation and Optical activity}

Polarimetry [8] is the measurement and interpretation of the polarization of transverse waves, most notably electromagnetic waves, such as radio waves and light. Typically polarimetry is conducted on electromagnetic waves that have traveled through or reflected, refracted or diffracted from some material or object in order to characterize that object. A polarimeter is the basic scientific instrument used to make these measurements.

Optically active material, such as solutions of chiral molecules, often exhibit circular birefringence. Circular birefringence causes rotation of the polarization of planepolarized light as it passes through the sample. A simple polarimeter to measure this rotation consists of a long tube with flat glass ends, into which the sample is placed. At each end of the tube is a Nicol prism or other polarizer. Light is shone through the tube and the prism at the other end, attached to an eyepiece, is rotated until all light is shut off. The angle of rotation is then read off on a scale. The specific rotation of the sample may then be calculated. Temperature can affect the rotation of light; therefore temperature should be accounted for in the calculation.

Thus, optical activity of any sample is a measure of the ability of such sample, in the form of solution, to rotate the plane of polarization, if it is placed in the path of plane-polarized light. Any substance is said to be optically active material if it rotates the plane of the polarized light, and vice versa. A substance is called as a non-optically active material if it does not rotate the plane of the polarized light.

\section{Dextrorotation and Levorotation}

Transmission of radiation in any optically isotropic media, such as homogeneous gases, liquid or solids that crystallize in cubic form is different compared to that in anisotropic media. In optically media, radiation will be transmitted at equal velocities in all directions, regardless of the polarization of the radiation. In any anisotropic media, such as non-cubic crystals, on the other hand, radiation may be transmitted at different velocities, depending upon the angular relationship between the plane of polarization and a given axis of the crystal. $\alpha$-Alanine, the sample under investigation in this work, is an anisotropic medium, since it belongs to orthorhombic crystal system.

Behavior of plane-polarized radiation on passage through an optically anisotropic medium can be treated as the interfer-

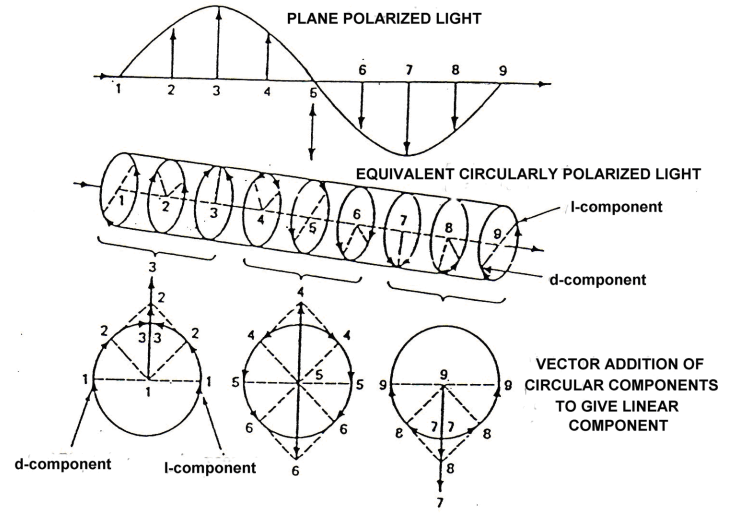

FIG. 2: Equivalence between circular and plane-polarized light: a) Plane-polarized light, b) Equivalent circularly polarized light, c) Vector addition of circular components to give linear components

ence product of two coherent circular rays of equal amplitude that rotate in opposite directions. Those two rays or two vectors are d- (dextrorotation), referring to clockwise rotation, and $\ell$ - (levorotation), referring to anticlockwise rotation, as beam approaches the observer.

Each of the two rotating vectors, $\mathrm{d}$ - and $\ell$ - components describes a helical path around the axis of propagation of the beam. The vectors for the $\mathrm{d}$ - and $\ell$-circular components are then added in order to produce equivalent vectors of a planepolarized beam. This explanation is shown in Figure 2.

Rotation of the equivalent vector of plane-polarized light by an optically active substance can be explained if one assumed that the rates of propagation of the $\mathrm{d}$ - and $\ell$ - circular components are different. Therefore, the refractive index of the substance with respect to the d-radiation $\left(\mathrm{n}_{d}\right)$ is different from that for the $\ell$-radiation $\left(\mathrm{n}_{\ell}\right)$. As a result, an optically active substance is anisotropic with respect to circularly polarized light. The two circular components, however, are no longer coherent in anisotropic medium and they cannot interfere until they reach an isotropic medium again.

For any system in which $\left(\mathrm{n}_{\ell}\right)<\left(\mathrm{n}_{d}\right)$, rotation of the equivalent vector of the plane-polarized light within the experimental setting of polarimeter is explained in Figure-3. (a) A polarimeter is set to allow the polarized light in a vertical plane with the circular $\mathrm{d}$ - and $\ell$-components rotating at equal velocities. Hence, the emerging plane-polarized light from the polarizer is in the vertical plane as shown. (b) After emerging any anisotropic medium (on the passage of the polarized light through the tube containing of optically active solution), the rate of propagation of the d-component is slowed more than that of the $\ell$-component, due to $\left(\mathrm{n}_{\ell}\right)<\left(\mathrm{n}_{d}\right)$. Therefore, at some locations in the medium, the $d$ - vector lags behind the $\ell$-vectors. Suppose at this point the two rays could interfere, the resultant would still be a plane, but one that was rotated from the vertical. (c) At a further point, the d-component would still be further retarded and a greater rotation would result. (d) Eventually, after emerging from the tube and reaching the analyzer, retardation has been such that the resultant plane is now horizontal. The final result is that the d- and 


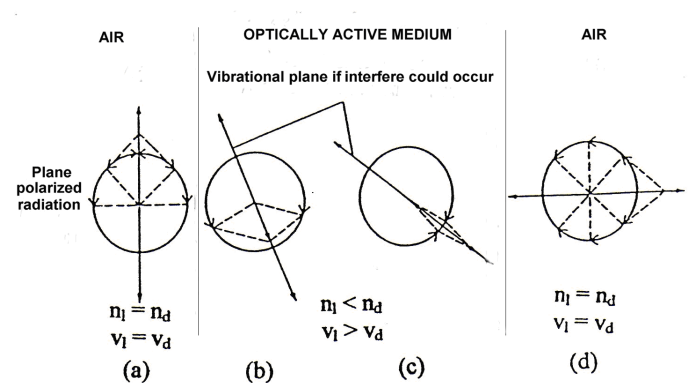

FIG. 3: Rotation of equivalent vector of plane-polarized light in any medium that has $\left(\mathrm{n}_{\ell}\right)<\left(\mathrm{n}_{d}\right)$ [9]. Detailed explanations on parts (a) to (d) are given in the text above.

$\ell$-components propagate in air at the same rates as in the isotropic air medium and interference of the coherent rays occurs.

\section{EXPERIMENT}

\section{Sample Preparation}

About 1.0 gram of each L-, D- and DL- $\alpha$-alanine powder samples (Merck), $10 \mathrm{ml}$ of $\mathrm{H} 2 \mathrm{O}$ and $2 \mathrm{ml}$ (about 2-mole $\mathrm{dm}^{-1}$ ) of chloride acid $(\mathrm{HCl})$ were weighed to make up $8.3 \%$ concentration (c) or ( $=8.3$ gram ml $\left.^{-1}\right)$ of L-, D- and DL- $\alpha$ Alanine solutions. Weighting of those samples was conducted at room temperature by using a Mettler AE-200 balance. 2mole $\mathrm{dm}^{-3}$ of $\mathrm{HCl}$ was added into the solution in order to measure rotation of the plane-polarized light significantly.

\section{Measurement}

A Perkin-Elmer Model 141 polarimeter with a wavelength of 589-nm (D-line) and that was equipped with a digital counter was utilized in this experiment to measure the specific rotations from the three $\alpha$-alanine solutions above. A standard sample cell made of a special optical glass for the visible range, and of quartz for the UV range into which a solution was placed, was also utilized. The path length and the sample cell volume are $100 \mathrm{~mm}$ and $5 \mathrm{ml}$, respectively. The experiment was run at room temperature $\left(25^{\circ} \mathrm{C}\right)$.

$$
\text { The observed rotation }=[\alpha]_{\lambda}^{t^{\circ}}
$$

Equation (1) is the rotation of the digital counter (either clockwise or anti clockwise) to express the number of degrees rotation of the analyzer in order to allow the plane-polarized light to go through to the observer from the analyzer. The specific rotation is then defined as the observed rotation per unit of concentration of the solution and length of the light path in the solution. Therefore,

$$
\text { The specific rotation }=\frac{[\alpha]_{\lambda}^{t^{\circ}}}{c \ell}=\frac{100 \alpha}{c \ell}
$$

Where $\mathrm{t}^{\circ}=$ temperature of the solution (in this experiment, $\mathrm{t}$ $\left.=25^{\circ}\right), \lambda=$ wavelength of Sodium light (D-line, $589 \mathrm{~nm}$ ), $\mathrm{c}=$ concentration of solution, $\ell=$ length of the light path in the solution (dm) and $\alpha=$ the observed rotation in degrees, either positive (clockwise) or negative (anti clockwise) from the observer point a view.

\section{RESULTS AND DISCUSSION}

Table-I lists the observed rotation of the digital counter of the Perkin Elmer Model 141 polarimeter with respect to the observer's point a view due to three solutions of L-, D- and DL- $\alpha$-alanine samples. Their specific rotation data were also listed.

\section{L- $\alpha$-alanine}

As can be seen from the Table-I above that L- $\alpha$-alanine solution rotated the digital counter of the polarimeter clockwise by $+(0.2880 \pm 10-1)^{\circ}$ and the specific rotation of the plane-polarized light due to this rotation is $+(3.4700 \pm 10-1)$ $/ \mathrm{dm}$ gram $\mathrm{ml}^{-1}$. As a comparison, Table-II lists the specific rotation of L- $\alpha$-alanine solution provided by Aldrich, Catalog Handbook of Fine Chemicals [10] and Lide et al.[11] that were measured in various experimental conditions, such as temperatures, concentrations and the solvent used.

As listed in Table-II, the value of the specific rotation of the plane-polarized light due to L- $\alpha$-alanine solution is different from one system to the others. This is because the experimental conditions for each system, such as the concentration, temperature of the solution and the solvent used to make up the solution, either $\mathrm{H}_{2} \mathrm{O}$ or $\mathrm{HCl}$, are also different to each other. As explained previously, given the experimental conditions, the more acidic the solution, the greater the rotation of the plane-polarized light, as proven in Table-II. (Detail discussion on the usage of chloride acid in these solutions to speed-up the rotation of a plane-polarized light is given after the discussion of the DL- $\alpha$-alanine result).

Referring back to data in Tables-I and Table-II. The specific rotation value due to $\mathrm{L}-\alpha$-alanine solution obtained in this experiment is $(+3.4700)^{\circ} / \mathrm{dm}_{\text {gram ml}}{ }^{-1}$ while that in Table-II is $(+2.8)^{\circ} / \mathrm{dm}_{\text {gram } \mathrm{ml}^{-1}}$, both values are not exactly the same, but they are still in a good agreement, (in terms of the experimental conditions), indicates that the result obtained in this experiment is good. The discrepancy appear is believed due to different concentration between both solutions. Furthermore, all data provided in Table-II, as verified in this work, established that L- $\alpha$-alanine solution is an optically active substance because it possesses the ability to rotate the plane-polarized light clockwise or positive as seen by the observer. Such substance called, as explained above, as a dextrorotatory sample.

\section{D- $\alpha$-alanine}

As for the L- $\alpha$-alanine solution, rotation of the planepolarized light was also observed in the presence of D- $\alpha$ alanine solution in the sample cell of the polarimeter (refers 
TABLE I: The observed and the specific rotation of the polarimeter (as seen by the observer) due to solutions of three $\alpha$-alanine isomers

\begin{tabular}{cccc}
\hline \hline No. & Solution of & $\begin{array}{c}\text { The observed rotation } \\
{[\alpha]_{\lambda}^{t^{\circ}}\left( \pm 10^{-1}\right)^{\circ}}\end{array}$ & {$[\alpha]_{\lambda}^{t^{\circ}} / c \ell\left( \pm 10^{-1}\right)^{\circ} / \mathrm{dm} \mathrm{gram} \mathrm{ml}^{-1}$} \\
\hline 1 & L- $\alpha$-alanine & +0.2880 & +3.4700 \\
2 & D- $\alpha$-alanine & -0.3050 & -3.6750 \\
3 & DL- $\alpha$-alanine & -0.0001 & -0.0012 \\
\hline \hline
\end{tabular}

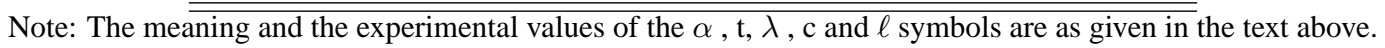

TABLE II: The specific rotation of L- $\alpha$-alanine solution measured at various temperature, concentrations and solvent used Aldrich Catalog[10] and Lide et al.[11]

\begin{tabular}{|c|c|c|c|}
\hline No. & $\begin{array}{l}\mathrm{L}_{\alpha} \text {-alanine } \\
\text { Solution of }\end{array}$ & $\begin{array}{l}\text { The specific rotation } \\
{[\alpha]_{\lambda}^{\circ}\left(\mathrm{dm} \text { gram } \mathrm{ml}^{-1}\right)} \\
\end{array}$ & $\begin{array}{c}\text { Concentration of the solution; } \\
\text { The solvent used }\end{array}$ \\
\hline 1 & $99 \%$ & {$[\alpha]_{D}^{20}=+14$} & $\mathrm{c}=6 ; 1$ mole $\mathrm{dm}^{-3} \mathrm{HCl}$ \\
\hline 2 & ${ }^{15} N, 98$ atom\% & {$[\alpha]_{D}^{25}=+15$} & $\mathrm{c}=0.6 ; 1$ mole $\mathrm{dm}^{-3} \mathrm{HCl}$ \\
\hline 3 & $99 \%$ & {$[\alpha]_{D}^{15}=+14.7$} & $\mathrm{c}=5.8 ; \mathrm{H}_{2} \mathrm{O}$ \\
\hline 4 & - & {$[\alpha]_{D}^{25}=+2.8$} & $\mathrm{c}=6 ; \mathrm{H}_{2} \mathrm{O}$ \\
\hline
\end{tabular}

to Table-I). The observed value is $-\left(0.3050 \pm 10^{-1}\right)^{\circ}$ in an anticlockwise direction as seen by the observer. This value gives the specific rotation of $-\left(3.6750 \pm 10^{-1}\right)^{\circ} / \mathrm{dm}$ gram $\mathrm{ml}^{-1}$. Meanwhile, the data published by Aldrich Catalog[10] and Lide et al[11] are as given in Table-III.

As shown in Table-III, all the solvent used to make up D$\alpha$-alanine solution was chloride acid $(\mathrm{HCl})$ and no particular solution in the $\mathrm{H}_{2} \mathrm{O}$ solvent was listed. Since in this experiment all substances were dissolved in $\mathrm{H}_{2} \mathrm{O}$ liquid (and just added by a small fraction of $\mathrm{HCl}$ ), therefore no exact comparison on the specific rotation values between data in Table-III above and that was obtained in this experiment can be carried out. Further, as seen in Tables 2-3, that at the same experimental conditions, the specific rotation of the L- and D- $\alpha$ alanine solution are the same, they differs only in sign $(+14$ and 14.2$)^{\circ} / \mathrm{dm}$ gram $\mathrm{ml}-1$, respectively. This indicates the existence of equal numbers of L- and D- $\alpha$-alanine molecules in each substance. This indication also appears in this experiment, but the values are smaller, that is, $(+3.4700$ and $3.6750)^{\circ} / \mathrm{dm}$ gram $\mathrm{ml}-1$. (See Table-I). The values given in the Table-III are much greater than that of in this work. This difference, as discussed above, arises from different solvent used to make up solutions. Again, all data presented here established the property of optic activity of D- $\alpha$-alanine substance. Further, since this substance has the ability to rotate the plane-polarized light anti clockwise or negative with respect to the observer, so this substance called as a levorotatory sample, as described above.

\section{DL- $\alpha$-alanine}

As for L- and D- $\alpha$-alanine solutions, optical activity of DL$\alpha$-alanine solution in the sample cell of the polarimeter was also obtained (refers back to Table-I). Rotation of the digital counter, however, was very small, $(-) 0.0001^{\circ}$. This is more likely due to electrical noise during the experiment rather than due to different rates of propagation between the circularly polarized components of the light. Thus, no rotation of the plane-polarized light was observed from the DL- $\alpha$-alanine solution. Again, this might be due to the existence of similar number of the D- and L- molecules in the DL-solution. Each molecule rotates the plane-polarized light at almost the same rates but in the opposite directions. Therefore, their rotations cancel each other. No specific rotation data on DL-solution is published, indicating this is a non-optically active substance, as verified in this experiment.

\section{Adding of chloride acid in solution}

In this experiment, significant observed rotations were achieved by introducing a rotational shift, positive or negative, in the direction of the molecular rotation in solution. This rotational shift arises from ionization or addition of acid to an aqueous solution of an optically active $\alpha$-amino acid. The main idea of the rotational shift induced by ionization is based on the configuration of the $\alpha$-asymmetric carbon atom. This method relies, in essence, on the relative contributions of the carboxylate $\left(\mathrm{COO}^{-}\right)$and carboxyl $(\mathrm{COOH})$ groups to the optical rotatory power of the molecules. The $(\mathrm{COOH})$ group in an acidic aqueous solution has a greater contribution to the rotation in the direction of the molecular rotation than a $\left(\mathrm{COO}^{-}\right)$ group that is found in water $\left(\mathrm{H}_{2} \mathrm{O}\right)$. Therefore, in this experiment, an $\alpha$-alanine chloride solution was made in order to obtain significant rotations of the plane-polarized light. This method was followed due to very small rotation of the digital counter of the analyzer resulted from $\alpha$-alanine solution in $\mathrm{H} 2 \mathrm{O}$ without acid addition.

An acid[9] is any hydrogen containing substance that capable to produce hydronium ion $\left(\mathrm{H}_{3} \mathrm{O}^{+}\right)$in aqueous solution by donating protons to solvent molecules. Therefore, the reaction between $\mathrm{HCl}$ and $\mathrm{H}_{2} \mathrm{O}$ is:

$$
\mathrm{HCl}(a q)+\mathrm{H}_{2} \mathrm{O}(\mathrm{liq}) \rightarrow \mathrm{H}_{3} \mathrm{O}^{+}(a q)+\mathrm{Cl}^{-}(a q)
$$

The increase in concentration of $\mathrm{H}_{3} \mathrm{O}^{+}$in Equation (3) is considerable when $\mathrm{HCl}$ is added to $\mathrm{H}_{2} \mathrm{O}$. At the equilibrium 
TABLE III: The specific rotation of D- $\alpha$-alanine solution measured at various temperature, concentrations and solvent. The data was obtained from the Aldrich Catalog [10] and Lide et al.[11]rotation of L- $\alpha$-alanine solution measured at various temperature, concentrations and solvent used Aldrich Catalog[10] and Lide et al.[11]

\begin{tabular}{|c|c|c|c|}
\hline No. & $\begin{array}{l}\mathrm{D}_{\alpha} \text {-alanine } \\
\text { Solution of } \\
\end{array}$ & $\begin{array}{l}\text { The specific rotation } \\
{[\alpha]_{\lambda}^{t^{\circ}}\left(\mathrm{dm} \text { gram } \mathrm{ml}^{-1}\right)}\end{array}$ & $\begin{array}{c}\text { Concentration of the solution } \\
\text { and the solvent used }\end{array}$ \\
\hline 1 & $99 \%$ & {$[\alpha]_{D}^{20}=-14.2$} & $\mathrm{c}=6 ; 1$ mole $\mathrm{dm}^{-3} \mathrm{HCl}$ \\
\hline 2 & - & {$[\alpha]_{D}^{25}=-13.6$} & $\mathrm{c}=1 ; 6$ mole $\mathrm{dm}^{-3} \mathrm{HCl}$ \\
\hline
\end{tabular}

condition, total concentration of acid $\left(\mathrm{K}_{a}\right)$ is,

$$
K a=[H A]+\left[A^{-}\right]
$$

Whereas acid ionization constant $\left(\mathrm{k}_{a}\right)$ is,

$$
k_{a}=\frac{\left[H^{+}\right]\left[A^{-}\right]}{[H A]}
$$

Since $\mathrm{HCl}$ is a strong acid, thus $\left[\mathrm{Cl}^{-}\right]$in Equations (4) and (5) is much greater than [HCl]. This leads to bigger values of total concentration of this acid $\left(\mathrm{K}_{a}\right)$ and acid ionization constant $\left(\mathrm{k}_{a}\right)$. Adding $\mathrm{HCl}$ into an aqueous solution changes the acidity $(\mathrm{pH})$ of the solution. The newly acidified $\alpha$-alanine solution now becomes more acid because $\left[\mathrm{H}_{3} \mathrm{O}^{+}\right]$is considerably increased. In other words, the $\mathrm{pH}$ value is now lower compared to the previous solution $\left(\mathrm{H}_{2} \mathrm{O}\right)$. Thus, in $\alpha$-alanine chloride solution, the $(\mathrm{COOH})$ group is formed instead of $\left(\mathrm{COO}^{-}\right)$. Finally, significant rotations of plane-polarized light in the direction of the molecular rotation can be observed through the digital counter of the polarimeter. These what happened in this work.

Moreover, as this method can also be considered as a qualitative application of the rule of optical superposition, therefore, acidification of an aqueous solution of L-amino acid gives rise to more positive of molecular rotation and hence polarization. Likewise, if the same treatment is applied to a Damino acid leads to more negative of molecular rotation and hence polarization.

\section{$\alpha$-Alanine: An asymmetric compound}

As discussed above, $\alpha$-alanine molecules display an optical rotatory ability, that is the ability of the molecules to rotate the plane of the polarized light, either to the left or to the right. This ability can be attributed to the fact that $\alpha$-carbon atom of this molecule attaches to four different groups (see Figure1a). Due to this attachment, $\alpha$-alanine is categorized as an asymmetric molecule, because it does not possess complete symmetry when viewed from a purely geometrical standpoint. The asymmetric structure means that the two structures relate to each other as an object to its mirror image or the right hand to the left (see Figures-1b and 4). As a consequence of this structural variation, two tetrahedral arrangements of the groups about the asymmetric $\alpha$-carbon atom attached, that are proposed for, respectively, the D- and L- $\alpha$-alanine structures, also relate to each other as an object to its mirror image. Thus, if equal amounts of these mirror image molecules (D- and L) are put together, the result is DL- $\alpha$-alanine compound that

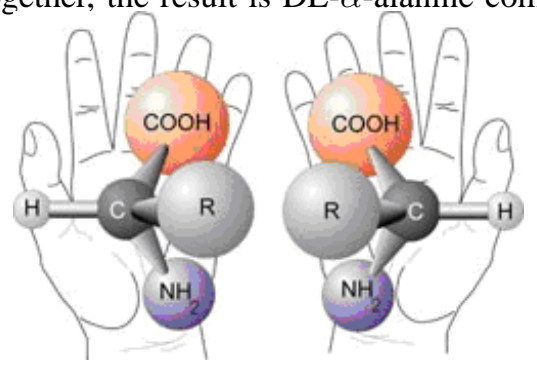

FIG. 4: Asymmetric structure of $\alpha$-alanine, that is, two structures (Dand L-) that relate to each other as an object to its mirror image, or the right hand to the left. These structures results in opposite direction of optical activity property in D- and L- $\alpha$-alanine.

has neutral optical effect due to the cancellation from the constituent isomer's optical effect, as verified in this experiment.

\section{CONCLUSION}

The properties of optical activity of three isomers of $\alpha$ alanine (D-, L- and DL- $\alpha$-alanine) are verified in this work. It was obtained that both D- and L- $\alpha$-alanine are optically active substances. However, DL- $\alpha$-alanine is a non-optically active one. The existence of similar number of the D- and L- molecules in the DL-solution leads to this. D- and L- $\alpha$ alanine are therefore classified as dextrorotatory and levorotatory sample, respectively. That classification is due to asymmetric structure of the system.
[1] Panta, et al., Proc. 4th Working Radiation Interactions, Liepzig, 365-69,1987.
[2] Panta, et al., Appl. Radiat. Isot., 40, 10-12, 971-75 (1989).

[3] Regulla, D. F. and Deffner U., Appl. Radiat. Isot., 33, 1101-14 
(1982).

[4] Sagstuen, E. et al., Nucleonika, 42, 353 (1997)

[5] Sagstuen, E. et al., J, Phys. Chem. 101, 50, $9763-72$ (1997)

[6] IUPAC-IUBMB Joint Commission on Biochemical Nomenclature, Nomenclature and Symbolism for Amino Acids and Peptides, (www.chem.qmul.qc.uk/iupac/AminoAcid/). Recommendations on Organic \& Biochemical Nomenclature, Symbols \& Terminology etc. Retrieved on 2007-05-17.

[7] Kendall, E. C.; McKenzie, B. F. Alanine, Organic Syntheses, 1, 21, (www.orgsyn.org/orgsyn/pdfs/CV1P0021.pdf). Retrieved on
2007-01-23 (1941).

[8] Polarimetry, Dextrorotation and Levorotation, Alanine (http://en.wikipedia.org/). Retrieved on 2008-05-10.

[9] Solomon, T. W.; Graham and Graig B. F., Organic Chemistry (8th Hoboken: John Wiley and Sons, Inc, 2004)

[10] Aldrich: Catalog Handbook of Fine Chemicals, Wisconsin, USA, Aldrich Chemical Company, Inc., 2000.

[11] Lide, D. R. and Milne, G. W. A., Handbook of Data on Organic Compounds,(3rd Edition, Vol. 1, CRC Press. 1994). 